\title{
The Eye of a Mathematical Physicist
}

\section{Journal Article}

Author(s):

Hepp, Klaus

Publication date:

2009

Permanent link:

https://doi.org/10.3929/ethz-b-000422856

Rights / license:

In Copyright - Non-Commercial Use Permitted

Originally published in:

Journal of Statistical Physics 134, https://doi.org/10.1007/s10955-009-9719-z 


\title{
The Eye of a Mathematical Physicist
}

\author{
Klaus Hepp
}

Received: 9 October 2008 / Accepted: 1 March 2009 / Published online: 25 March 2009

(C) Springer Science+Business Media, LLC 2009

\begin{abstract}
In this essay we are searching for neural correlates of 'doing mathematical physics'. We introduce a toy model of a mathematical physicist, a brain connected with the outside world only by vision and saccadic eye movements and interacting with a computer screen. First, we describe the neuroanatomy of the visuo-saccadic system and Listing's law, which binds saccades and the optics of the eye. Then we explain space-time transformations in the superior colliculus, the performance of a canonical cortical circuit in the frontal eye field and finally the recurrent interaction of both areas, which leads to a coherent percept of space in spite of saccades. This sets the stage in the brain for doing mathematical physics, which is analyzed in simple examples.
\end{abstract}

Keywords Brain mathematics · Computational neuroscience · Eye movements · Vision

\section{Introduction}

Mathematicians are very curious as to how their brains function when they are 'doing' mathematics, probably because more than any other intellectual community they depend strongly on the operational characteristics of their 'mental organ'. A number of interesting books have recently been written on this subject, mostly from the introspective point of view $[10,12,18,19,86,101]$. With the emergence of the neurosciences there is hope for new answers on two different levels:

The most ambitious program is the 'Blue Brain' initiative to simulate the brain 'isomorphically' in a gigantic computer, to read out the laws of cognitive operations, and to model the mind of, say, a mathematician [66]. On the other hand, neuropsychology has given, based on non-invasive mappings (e.g. functional magnetic resonance imaging (fMRI) and magnetoencephalography (MEG) of normal and brain-lesioned humans), a macroscopic picture

Dedicated to Jürg Fröhlich for his 60th anniversary.

K. Hepp (殴

Institute for Theoretical Physics, ETHZ, CH 8093 Zürich, Switzerland

e-mail: khepp@phys.ethz.ch 
of brain areas involved in cognition, without having the space-time resolution to measure the activity of single neurons. Here, however, one can use the data from lower primates, mainly macaque monkeys as well as cats and rodents, with rigorously controlled anatomy, physiology and behaviour, and more flexible analytical tools in 'Mini Blue Brain' (MBB) models.

As a traveller between mathematical physics and neurobiology I want to present some material from my own limited experience, with immodest references to the work of my collaborators in neurology and neuroinformatics. I will refrain from producing images, but I will refer extensively to the original literature, often to later elaborations than to priority. For links to neurobiology, I have used many anatomical terms which are collected in Sect. 2 or printed in Italics, when they first occur. I hope that a few idle mathematical physicists will look up part of the quoted literature and get involved with one of the many unsolved theoretical problems in brain research.

My question is: What are neural correlates of doing mathematical physics?

The neurosciences are exploding with factual data, e.g. with more than 10.000 posters or talks at every annual meeting of the Society for Neuroscience. One of the best studied topics in systems neuroscience is the relation between vision and rapid eye movements (saccades) in macaque and man. Following Goethe ('Wär' nicht das Auge sonnenhaft, wie könnten wir das Licht erblicken?'-were not the eye like the sun, how could we see the light?) I expect to reach here a more precise form of my question and hopefully some answers. My image of a mathematical physicist is inspired by Descartes' 'Traité de l'homme' (Fig. 33 in [23]) and will be modernized in Sect. 2. Two of the basic problems to be treated have already been posed by the great medical and mathematical physicist von Helmholtz [146], based on clever psychophysical experiments and nontrivial mathematics:

- The eye ball is a center-fixed sphere with its configuration space the 3-dimensional rotation group $S O$ (3), while the retina lives on a 2-sphere $S_{2}$. What is the optimal mapping between $S O(3)$ and $S_{2}$ for vision and eye movements?

- How does the brain provide stability of vision despite of saccades?

Both questions are closely related. The first, leading to Listing's law, is about the optimal choice of a visuo-motor 'program'. The second is about its use. In good numerical approximation, saccades redirect the direction of sight in $50 \mathrm{~ms}$ with a speed up to $1000 \mathrm{deg} / \mathrm{sec}$ to acquire a salient target with the fovea. The latter has a radius of $1 \mathrm{deg}$. A mathematician scans an interesting paper in a perifoveal range of $20 \mathrm{deg}$. He often makes three saccades per second and maintains a stable view of a text covered with formulas within the panoramic background of 100 deg diameter. The saccade-induced image motion over the retina would be intolerable for his work. Equally perturbing would be the blanking out of the scrutinized formula and its reappearance elsewhere for its slow semantic analysis, which can take a second. Visual stability is an aspect of translation invariance of vision, despite of saccades, and more generally of internal models, a subject today under intensive study.

Of course, my essay will deal only with very special functionalities of the brain related to doing mathematical physics, more on the action than on the perception side. However, my understanding is modest. Fortunately a working mathematician is not climbing in the trees and picking fruits, where many more sensorimotor degrees of freedom are activated. We shall see that our toy model will give us some insights of more general interest.

In Sect. 2 we shall give a concise description on the neuroanatomy of the visuo-saccadic system. In Sect. 3 we will introduce Listing's law for binding the kinematics of saccades and the optics of the eye. Section 4 will deal with methodology. In Sect. 5 we will discuss 
operations of the superior colliculus, where vision and saccades intertwine on the neural level. In Sect. 6 the interaction between vision, attention and saccades will be outlined in a neocortical circuit MBB model of the frontal eye field. In Sect. 7 we shall discuss new experimental data on how visual stability is implemented between neocortex and superior colliculus, leading to the concept of translation invariance, which in Sect. 8 will give us some insights into doing mathematical physics in the brain.

\section{Blueprint of a Mathematical Physicist}

In this section I will introduce a toy model of a mathematical physicist (M $\Phi$, not of Jürg), as I see them daily through the glass walls in our beautiful 'Branco Weiss Information Science Laboratory'. This allows me later to use neurophysiological terms as a bridge to the literature. All numbers are approximate. System neuroscience relies heavily on functional neuroanatomy. Please consult Wikipedia (www.wikipedia.org/) on 'brain' and Google for beautiful pictures at 'brain anatomy'.

$\mathrm{M} \Phi$ sits with his(/her) head upright and stationary in front of a computer screen at a distance of $80 \mathrm{~cm}$. The diameter of the screen is $40 \mathrm{~cm}$ and the distance between his two eyes is $8 \mathrm{~cm}$. With the eyes slightly converged he fixates or scans text by saccades within a 'periprimary range' of $20 \mathrm{deg}$ radius. Many studies of higher brain functions in man and monkey are made in such a limited workspace. Between fixation and saccades, $M \Phi$ generates highlevel associations between visual inputs from the screen, which he can modify, and which sometimes lead to illuminating 'insights'. In this minimal model we will not consider M $\Phi$ 's arm moving a mouse. In principle, the computer behind the screen could interact with $М \Phi$ using a code of saccade- and (eyelid) blink-patterns, which can be non-invasively recorded by an eye tracker. In a sense we have reduced $\mathrm{M} \Phi$ to a highly motivated intelligent human, connected to the world only by his visuo-saccadic system, as in the late J.-D. Bauby [5]. We will often analyze his brain using data from the macaque monkey (MAC), his junior fellow.

$M \Phi$ 's eye balls are to a good approximation center-fixed spheres of $10 \mathrm{~mm}$ radius. The action of six muscles can rotate each eye about any axis. Inside the eye-ball is the retina (RET), on which the external world is optically mapped. The photoreceptors (cones, the rods are bleached during day-light) are densest in the center of the fovea, with about 200.000 cones per $\mathrm{mm}^{2}$, and fall off very rapidly (half-maximum at $.15 \mathrm{~mm}$, or $.5 \mathrm{deg}$ ) and reach an asymptotic density of 2000 cones $/ \mathrm{mm}^{2}$ at $15 \mathrm{deg}$ [17].

When the head is stationary and upright, $\mathrm{M} \Phi$ can use, in a somewhat fuzzy distinction, four different conjugate oculomotor 'programs': three two dimensional (2D) eye movement programs, fixation, saccades and smooth pursuit (see Sect. 3) and a 3D (retinal image) stabilization program. Superimposed on them is convergence, i.e. eye movements in depth. With small constant convergence and no passive head movements in a stationary visual surround MФ's eye movements reduce to fixation, saccades and smooth pursuit. Since the latter can be approximated by saccades, we will not need smooth pursuit in our toy model. Note that saccades can be volontary, but can also be an automatic 'visual grasp reflex'.

$\mathrm{M} \Phi$ 's central nervous system (CNS) is shaped like a hammer with neocortex (NC) the (hammer-)head and the brainstem (BS) and spinal cord (SP) the shaft. The NC is divided by the central sulcus (CS) into a frontal and a posterior part. The BS is the rostral (upper) part of the shaft and the SP the caudal (lower) part. We are not interested in M $\Phi$ 's somatic motor system and we will not characterize it further. The junction between NC and BS is formed by the thalamus $(\mathrm{TH})$ and superior colliculus (SC). There are 'struts' called basal ganglia (BG), hippocampus (HI) and cerebellum (CB), about which I have little to say. In the front are the two big eyes, where the RET output enters the CNS without feedback. 
All anatomical structures of the CNS are mirror-symmetric, connected by many nerve fibers, with a lateral (outside) and medial (inside) surface. The upper surface of the head is called dorsal (back-sided), the lower ventral (belly-sided). The SC has a visual (visSC) and motor (motSC) layer and the TH is subdivided into compartments, among others the lateral geniculate (LG), pulvinar (PUL) and mediodorsal (MD) parts. In this essay we will attach functions to these structures.

M $\Phi$ 's (oculomotor) brainstem is a cylinder with two compartments: rostral the verticaltorsional oculomotor area (VT) and caudal the horizontal-convergence oculomotor area (HC). In VT are the motoneurons (MN), the integrator (BTN) and the saccadic burst neurons ( $\mathrm{SBN}$ ) of the vertical-torsional eye muscles (SR, IR, SO, IO), in HC are those of the horizonal-convergence muscles, lateral and medial rectus (LR, MR). The LR of the right (r) eye and the MR of the left (1) eye pull as agonists both eyes to the right during conjugate eye movements, and rLR and rMR are antagonists (more in Sect. 5).

M $\Phi$ 's neocortex is a large sheet of neurons of $2 \mathrm{~mm}$ thickness. In every $\mathrm{mm}^{3}$ there are about 50.000 neurons arranged in six horizontal layers. About $80 \%$ of the neurons are excitatory and $20 \%$ inhibitory (see [28]). One distinguishes local neuronal circuits, intraareal interactions and inter-areal cortico-cortical pathways. Excitatory inputs from subcortical structures enter layer 4 via the TH. The output to subcortical structures leaves NC by neurons from layer 5 and 6.

$\mathrm{M} \Phi$ 's needs many visual-, fixation- and saccade-related $\mathrm{NC}$ areas for his visuo-saccadic operations, among which only some will be mentioned. To the front are the prefrontal cortex $(\mathrm{PF})$ and closer to the CS the frontal eye field (FEF). In the CS lies the eye proprioception area. At the posterior end of $\mathrm{NC}$ is the visual cortex (VI) with the primary visual cortex V1 (or striate NC), then dorsal the intraparietal sulcus (IP) with the lateral (LIP) and ventral (VIP) areas, and ventral the inferotemporal cortex (IT). All these $\mathrm{NC}$ areas are bidirectionally connected. The pathway VI $\rightarrow \mathrm{IP} \rightarrow \mathrm{PF}$ is called the dorsal stream and VI $\rightarrow \mathrm{V} 4 \rightarrow \mathrm{IT} \rightarrow \mathrm{PF}$ the ventral stream, including the extrastriate area V4 in VI. Only FEF has a strong direct projection to motSC. Important are the input pathways $\operatorname{motSC} \rightarrow \mathrm{MD} \rightarrow$ FEF and motSC $\rightarrow$ PUL $\rightarrow$ LIP.

Exercise: draw the blueprint and keep it in your long-term memory.

\section{Listing's Law}

In this section we shall see that Helmholtz' first question has a simple answer from four different perspectives.

Listing's law states that, with the head stationary and the eyes fixating a distant target, the rotation axes between a head- and an eye-fixed coordinate system all lie in a plane orthogonal to the primary position (a head and eye forward direction). For fixation Listing's law was found by observing afterimages, and its mathematical formulation is by the Göttingen physicist JB Listing (1808-1882) and by H von Helmholtz (1821-1894). For saccades and fixation Listing's law was a striking observation for all neuro-ophthalmologists using the 3D magnetic search coil invented by Robinson [94]. For smooth pursuit Listing's law was shown to hold in humans by Tweed et al. [137] and in monkeys by Haslwanter et al. [44], where Figs. 1 and 2 illustrating Listing's law (recorded in MAC) characterize a М $Ф$ looking at his screen. We shall not describe here the generalization of Listing's law for convergence (see e.g. $[16,135])$.

For a mathematical discussion, let $\left\{\boldsymbol{h}_{x}, \boldsymbol{h}_{y}, \boldsymbol{h}_{z}\right\}$ be a head-fixed and $\left\{\boldsymbol{e}_{x}, \boldsymbol{e}_{y}, \boldsymbol{e}_{z}\right\}$ an eyefixed right-handed orthonormal coordinate system. For oculomotor applications $\boldsymbol{h}_{x}$ points 
forward in the midplane close to the center of the oculomotor range, $\boldsymbol{h}_{y}$ is directed leftward through the line through the center of both eyes, and $\boldsymbol{e}_{x}$ is along the direction of sight through the fovea. Both systems are connected by a $R \in S O(3)$ such that $\boldsymbol{e}_{k}=R \boldsymbol{h}_{k}=$ $\left(\boldsymbol{h}_{k} \cdot \boldsymbol{e}\right) \boldsymbol{e}+\sin \rho \boldsymbol{e} \times \boldsymbol{h}_{k}-\cos \rho \boldsymbol{e} \times\left(\boldsymbol{e} \times \boldsymbol{h}_{k}\right)$. By the right hand rule, $R=R(\varrho, \boldsymbol{e})=R(\boldsymbol{r})$ is characterized by its rotation axis $\boldsymbol{e}$ and angle $\varrho \geqslant 0$ or by its rotation (Euler-Rodrigues) vector $\boldsymbol{r}=\tan (\rho / 2) \boldsymbol{e}$. To the inverse $R^{-1}$ coresponds the rotation vector $-\boldsymbol{r}$, and to the product $R\left(\boldsymbol{r}_{1}\right) \cdot R\left(\boldsymbol{r}_{2}\right)$

$$
\boldsymbol{r}_{1} \circ \boldsymbol{r}_{2}=\left(\boldsymbol{r}_{1}+\boldsymbol{r}_{2}+\boldsymbol{r}_{2} \times \boldsymbol{r}_{1}\right) /\left(1-\boldsymbol{r}_{1} \cdot \boldsymbol{r}_{2}\right)
$$

For a trajectory $\boldsymbol{r}(t)$ on $S O(3)$ one has to distinguish between the coordinate velocity $\boldsymbol{v}(t)=$ $d \boldsymbol{r}(t) / d t$ and the angular velocity

$$
\boldsymbol{\omega}(t)=(\boldsymbol{v}(t)+\boldsymbol{r}(t) \times \boldsymbol{v}(t)) /\left(1+|\boldsymbol{r}(t)|^{2}\right)
$$

corresponding in matrix notation to $\Omega(t)=(d R(t) / d t) \cdot R(t)^{-1}$.

For every target direction relative to the head, characterized by a unit vector $s \in S_{2}$, there is a 1-parameter family of eye rotations $r(s)$, which foveate $s$ in the sense that $s$ will pass through the center of the eye and the fovea after a rotation with $\boldsymbol{r}(\boldsymbol{s}): R(\boldsymbol{r}(\boldsymbol{s})) \boldsymbol{h}_{x}=\boldsymbol{s}$. The simplest foveation is by a rotation $\boldsymbol{r}_{L}(\boldsymbol{s})=\boldsymbol{h}_{x} \times \boldsymbol{s} /\left(1+\boldsymbol{h}_{x} \cdot \boldsymbol{s}\right)$. If $\boldsymbol{s}=(a, b, c)^{T}$, then any other $\boldsymbol{r}(\boldsymbol{s})$ is a rotation $\boldsymbol{r}_{o}(x)=x \boldsymbol{h}_{x}$ about the $\boldsymbol{h}_{x}$-axis followed by $\boldsymbol{r}_{L}(\boldsymbol{s}): \boldsymbol{r}(\boldsymbol{s})=$ $\boldsymbol{r}_{L}(\boldsymbol{s}) \circ \boldsymbol{r}_{o}(x)$ and

$$
\boldsymbol{r}(\boldsymbol{s})=(x, y+x z, z-x y)^{T}, \quad y=-c /(1+a), z=b /(1+a), a=\left(1-b^{2}-c^{2}\right)^{1 / 2}
$$

Donders' law postulates that, when the head is upright and stationary, every direction $s$ in the oculomotor range is foveated with the eye in a unique position $\boldsymbol{r}(\boldsymbol{s})$, which varies smoothly with $s$. This implies that there exists a smooth 'Donders surface' $x(b, c)$ with $x(0,0)=0$, so that $\boldsymbol{r}(s)$ is defined by (3) with $x=x(b, c)$. Listing's law holds, if $x(b, c)$ is identically zero, i.e. if $\boldsymbol{r}(\boldsymbol{s})=\boldsymbol{r}_{L}(\boldsymbol{s})$ with $\boldsymbol{h}_{x}$ as primary position, and this defines the head-fixed Listing's plane

$$
\left\{\boldsymbol{r}: \boldsymbol{r} \cdot \boldsymbol{h}_{x}=0\right\}
$$

We shall always use Listing coordinates $\boldsymbol{r}=(T,-V, H)$ with $H, V$ and $T$ often called the horizontal, vertical and torsional angles of 'eye position'.

There are several justifications for Listing's law:

- Mechanical advantage: As a consequence of (3) $|\boldsymbol{r}(\boldsymbol{s})|^{2}=x^{2}+\left|\boldsymbol{r}_{L}(\boldsymbol{s})\right|^{2}$. Hence among all eye positions $\boldsymbol{r}(\boldsymbol{s})$ foveating $\boldsymbol{s}$, the Listing position $\boldsymbol{r}_{L}(\boldsymbol{s})$ has the smallest rotation angle. One could think that the minimization of $\varrho(s)=2$ atan $|\boldsymbol{r}(\boldsymbol{s})|$ for a given target direction $s$ is a consequence of the elasticity of the plant. However, when the eye is carried out of Listing's plane by a torsional head rotation [16] or by an imperfect saccade [144], it returns there only after the next saccade or a blink, and this needs neural control.

- Saccadic advantage: A set of eye positions connected by saccades which are geodesics in $S O(3)$ and do not violate Donders' law en route, is a Listing plane [49]. The physiological justification for this assumption is that geodesics of $S O(3)$ are fixed-axis rotations, and that in monkey and man the saccadic trajectories are generally well approximated by fixed-axis rotations. However, strongly curved saccades often occur for physiological reasons $[38,69,70]$.

- Visual advantage: This relies on a long, but inconclusive, variational principle by von Helmholtz [146] (see [50]). 
- Visuo-saccadic advantage: If Listing's law for saccades holds, then up to corrections $O\left(\operatorname{ran}^{3}\right)$, (ran: the range of eye movements considered) the relations between visual and saccadic coordinates are linear and commutative: for $\boldsymbol{r}_{1}$ and $\boldsymbol{r}_{2}$ in Listing's plane

$$
\boldsymbol{s}_{21}=2\left(\boldsymbol{r}_{2}-\boldsymbol{r}_{1}\right) \times \boldsymbol{h}_{x}, \quad \boldsymbol{s}_{32}=\boldsymbol{s}_{31}-\boldsymbol{s}_{21}
$$

The last advantage is for us most important, and the geometrical significance of (5) is best explained by its proof [51]: For 1D eye movements visuomotor integration is geometrically trivial. Modelling the surface of the eye as a 1-circle in a circular orbit, an angle $\Phi$ describes the position of the image of a distant visual target relative to the (center of the) fovea, and an angle $H$ the position of the fovea in the orbit. Let the eye- and head-fixed coordinate systems coincide in the forward direction. If the image of the target 1 in position $H_{1}$ falls on the fovea, then target 2 in position $H_{2}$ with 'retinal error' $\Phi_{21}$ can be foveated by the 'saccade vector' $\Delta H_{21}$

$$
\Phi_{21}=H_{2}-H_{1}=\Delta H_{21}, \quad \text { and } \quad \Phi_{32}=\Phi_{31}-\Phi_{21} \text { for three visual targets } 1,2,3
$$

We shall prove that these simple relations generalize to (5) up to small errors of $O\left(\operatorname{ran}^{3}\right)$, if Listing's law holds. Let $\boldsymbol{r}_{1}$ be a Listing position and $\left\{\boldsymbol{e}_{x 1}, \boldsymbol{e}_{y 1}, \mathbf{e}_{z 1}\right\}$ the eyefixed frame, rotated by $R\left(\boldsymbol{r}_{1}\right)$ out of $\left\{\boldsymbol{h}_{x}, \boldsymbol{h}_{y}, \boldsymbol{h}_{z}\right\}$ in primary position. We define $\boldsymbol{s}_{21}=$ $\left(0, \boldsymbol{e}_{x 2} \cdot \boldsymbol{e}_{y 1}, \boldsymbol{e}_{x 2} \cdot \boldsymbol{e}_{z 1}\right)$ as the retinal error between a target in the head-fixed direction $\boldsymbol{e}_{x 2}$ viewed from $\left\{\boldsymbol{e}_{x 1}, \boldsymbol{e}_{y 1}, \boldsymbol{e}_{z 1}\right\}$. Since $\sin \alpha=\alpha+O\left(\alpha^{3}\right)$, this is up to errors of $O\left(\operatorname{ran}^{3}\right)$ the correct generalization of polar coordinates on the retina (padded by 0$)$. Up to $O\left(\operatorname{ran}^{3}\right)$ one has for two eye-fixed frames rotated by $\boldsymbol{r}_{n}, n=1,2$ and for $k=x, y, z$

$$
\begin{aligned}
\boldsymbol{e}_{k n}=R\left(\boldsymbol{r}_{n}\right) \boldsymbol{h}_{k}=\boldsymbol{h}_{k} & +2 \boldsymbol{r}_{n} \times \boldsymbol{h}_{k}+2 \boldsymbol{r}_{n} \times\left(\boldsymbol{r}_{n} \times \boldsymbol{h}_{k}\right) \\
\Rightarrow \quad \boldsymbol{e}_{x 2} \cdot \boldsymbol{e}_{y 1} / 2= & \left(\boldsymbol{r}_{2} \times \boldsymbol{h}_{x}\right) \cdot \boldsymbol{h}_{y}+\left(\boldsymbol{r}_{1} \times \boldsymbol{h}_{y}\right) \cdot \boldsymbol{h}_{x}+\left(\boldsymbol{r}_{1} \cdot \boldsymbol{h}_{x}\right)\left(\boldsymbol{r}_{1} \cdot \boldsymbol{h}_{y}\right) \\
& +\left(\boldsymbol{r}_{2} \cdot \boldsymbol{h}_{x}\right)\left(\boldsymbol{r}_{2} \cdot \boldsymbol{h}_{y}\right)-2\left(\boldsymbol{r}_{1} \cdot \boldsymbol{h}_{x}\right)\left(\boldsymbol{r}_{2} \cdot \boldsymbol{h}_{y}\right)
\end{aligned}
$$

and a similar equation for $\boldsymbol{e}_{x 2} \cdot \boldsymbol{e}_{z 1} / 2$. The first two terms on the right hand side are as required by (5), and the others vanish, if $\boldsymbol{r}_{n}, n=1,2$, are in Listing's plane. As we shall discuss in Sect. 6, (5) is the geometrical basis for double-step saccades and for the updating of the visual representation of target 3 on the retina using the motor command $\boldsymbol{d}_{21}=\boldsymbol{r}_{2}-\boldsymbol{r}_{1}$ as internal feedback.

Numerically one finds that $\left|\boldsymbol{s}_{21}\right|$ differs from $\left|2 \boldsymbol{d}_{21}\right|$ maximally by less than $5 \%$, if for two Listing positions $\boldsymbol{r}_{1}$ and $\boldsymbol{r}_{2}$ the angles of $\boldsymbol{s}_{1}$ and $\boldsymbol{s}_{2}$ relative to $\boldsymbol{h}_{x}$ are less than $15 \mathrm{deg}$. In the periprimary range the motor error $\boldsymbol{q}_{21}$, the rotation vector of the eye rotation $R\left(\boldsymbol{r}_{2}\right) \cdot R\left(\boldsymbol{r}_{1}\right)^{-1}$ is well approximated by $\boldsymbol{r}_{2}-\boldsymbol{r}_{1}+\boldsymbol{r}_{1} \times \boldsymbol{r}_{2}$, with the vector product as a measure of the noncommutativity of eye rotations. The $O\left(\operatorname{ran}^{3}\right)$ corrections will probably never be observable in the firing patters of single neurons.

In Sect. 5 we shall study the most direct neural implementation of Listing's law in the superior colliculus, by binding two 2D neural maps of the periprimary visual field, of eye position and saccade vectors. This is a powerful tool for doing mathematical physics.

\section{Complexity of the Periphery}

It is obvious that one should proceed from the simple to the complex. For an ordinary human being and for MAC, the periphery V1 is important for vision, and the BS for generating 
the different oculomotor programs, mentioned in Sect. 2. However, these circuits have been developed for multiple purposes, among which 'doing $M \Phi$ ' is only a minor subtask. The periphery is extremely complex and has many idiosyncrasies inherited from lower mammals. D.A. Robinson, the Helmholtz of modern neuro-ophthalmology, has made a valiant effort to explain the oculomotor periphery by Newton-Euler mechanics and neural network theory [97]. Finally he realized that this is presently impossible [98]:

The main message of neural networks for the neurophysiologist is that the study of single neurons or neuron ensembles is unlikely to reveal the task in which they are participating or the contribution they are making to it. Conversely, even if one knows the function of a neural system, recording from single units is likely not to disclose how the function is being fulfilled by the signal processing of the neurons. A corollary is that being able to describe that function mathematically tells little about what to expect when recording from single neurons.

Unable to understand real neural networks at a synaptic or cellular level, we may be forced to wave our hands and say: 'Well, we do not know how it works in detail, but we do know that such and such a learning mechanism is at work here to allow synaptic weight changes that are compatible with observed learning, verified with models, and that's it'. This might be as close as we are ever going to get to explaining how a network does its thing. As a result, if we know the learning rules, we may have to accept the inexplicable nature of mature networks.

One encounters similar problems when starting at the visual periphery. The primary visual cortex (V1) has been intensely investigated for at least four decades since Hubel and Wiesel's studies of the visual receptive field and functional organization of single neurons in the cat's and in the monkey's visual cortex (see e.g. [54]). By definition, the visual receptive field (RF) of a neuron is the area of the visual field from which a response can be evoked. One of the main discoveries was that the responses of single neurons in the cortex could be related to distinct image properties, such as the local orientation of a contrast border.

The main advantage of studying a sensory area is that the stimulus properties are largely under the control of the experimenter, while in a motor area the output is under control of the subject. The main disadvantage is that it is unclear what aspects of the stimulus are used by the subject for behavior. With Hubel and Wiesel's discoveries this problem seemed to be circumvented, because parallel investigations in computer vision suggested a sequence of image transformations, which every efficient visual system had to implement [67]. Hubel and Wiesel discovered in V1 the first important steps from the symmetrical centre-surround RF in RET output cells and in the LG part of TH, to the simple cell (S) RF (with alternating subregions of light on- and off-response, responding best to oriented bars and edges), to the more translation-invariant RF of complex cells (C) (which are pooling over S-cells with similar orientation to make them insensitive to small changes in scale and location).

Today this computational approach to biological vision has reached a high level of performance, comparable to that of the best machine vision programs. Riesenhuber and Poggio [93] and Serre et al. [115] implemented a feedforward model, based on alternating stages of S-like summation and C-like maximalization operations, and Epshtein et al. [32] reach an even higher performance using a single bottom-up top-down cycle. However, the precise nature of the transformation at the first step from LG to V1 S-cells is still subject to interminable debates between modellers, which push experimenters to rare feats of sophistication. For C-cells, in spite of many very difficult experiments, no biophysically realistic neuronal network theory has been accepted. 
Olshausen and Fields [84] have analyzed how far we are in understanding V1 in natural vision. In terms of the simple formula: [fraction understood] $=$ [fraction of variance explained].[fraction of population recorded] and the estimate that roughly $40 \%$ of the populations in V1 has actually been recorded from and that $30 \%$ to $40 \%$ of the response variance of these neurons can be explained under natural conditions using the currently established models, they conclude that about $85 \%$ of V1 function has yet to be explained. They discuss new theories but see no hope for us to understand V1 in isolation.

My personal opinion is shaped by the landscape of Switzerland: there are summits in the clouds, which are difficult to climb, but beautiful walks in between. In the past decade neuroscience has made enormous progress, less on the BS than on the $\mathrm{NC}$, by giving up isolation, as suggested by Olshausen and Fields [84], and by studying the interaction of many areas in the appropriate idealization. This motivates my blueprint of $M \Phi$, and the rest of this essay.

\section{Space-Time Transformations}

In this section we will dive deeply into the brain at the junction between brainstem BS and neocortex NC. We will find a beautiful 'wetware' implementation of Listing's law in the superior colliculus SC.

The SC is a very intensively studied area in the macaque brain (see e.g. [123]). All cells in the upper layer visSC are visually responsive. Their visual RF vary as a function of excentricity in the visual field, they are perifoveally as small as .75 deg in diameter and up to $30 \mathrm{deg}$ diameter in the periphery. The visual field is topographically mapped on the surface of the visSC. Saccadic cells in the lower layer motSC discharge before saccades. They have movement fields, i.e. each neuron discharges maximally prior to saccades, characterized in 2D recordings by a particular vector $(\Delta H, \Delta V)$, regardless of initial eye position $(H, V)$. A gradient of response amplitude is observed across the movement field: Movements to the center of the movement field are preceeded by a vigorous discharge and less for movements deviating from the neuron's optimal vector. There is a topographical organization of movement fields. Some neurons that discharge prior to saccades also have visual receptive fields and are called visuo-saccadic cells. During visually evoked saccades they exhibit a visual burst with a latency of 70-100 ms (from the onset of a visual target to the peak of the response) and a well separated saccadic burst at about 120-160 ms to peak. There are also neurons in motSC which are purely visual and others, called quasivisual, where the visual response is not dependent on the activation of a particular part of RET. Inhibitory fixation neurons are found in the parafoveal region of the motor map [76].

For interpreting these data we need some qualitative understanding about the visuosaccadic input from RET to SC and output from SC to the MN of the eye muscles.

Output SC $\rightarrow$ MN: Robinson [95] recorded the firing patterns of LR MN and found a firing rate

$$
f(t-\tau)=\left[k \cdot\left(H(t)-H_{0}\right)+r \cdot d H(t) / d t\right]_{+}+\text {noise }
$$

as function of horizontal eye position. Here $H_{0}, r, k, \tau$ were constants and $[x]_{+}=\max \{x, 0\}$. The time delay $\tau \sim 6 \mathrm{~ms}$ and the time constant $r / k=T \sim 240 \mathrm{~ms}$ were approximately neuron independent. The position gain $k$ increased approximately linearly with the threshold $H_{0}$. For rLR $k$ was positive and increasing with $H_{0}$, for rMR, the antagonist, negative and decreasing with $H_{0}$, and mirror symmetrically for ILR and IMR. Robinson [97] guessed that 
by combining agonist and antagonist in a push-pull pair the total innervation $I_{\mathrm{MN}}$ of the horizontal muscles by MN should satisfy the linear differential equation

$$
\begin{aligned}
& I_{\mathrm{MN}}(t-\tau)=(H(t)+T \cdot d H(t) / d t) / a \\
& \tilde{H}(s)=a(s) \cdot \widetilde{I_{\mathrm{MN}}}(s), \quad a(s)=e^{\tau s} a /(1+T s)
\end{aligned}
$$

Here $a$ is a constant and $a(s)$ a transfer function in the language of Laplace transforms. Consider (8) for fixation for times $t<t_{1}$ and $t>t_{2}>t_{1}$ and a saccade to the right starting at $t_{1}$ and ending at $t_{2}$. Experimentally one found in a MN of the rLR above threshold that the firing rate is approximately constant at $f_{1}$ for $t<t_{1}-\tau$, that it 'bursts' beyond $f_{1}$ for $t_{1}-\tau<t<t_{2}-\tau$, and settles down at a higher level $f_{2} t>t_{2}-\tau$. This 'pulse-step' or 'burst-tonic' (BT) firing pattern of the MN of rLR for rightward saccades is not completely mirror symmetric for leftward saccades, because due to []$_{+}$in (8) a firing rate is never negative. Here the rLR MN has a 'pause step' firing pattern. The other three MN populations show similar patterns.

Close to the rLR there is a population of excitatory short lead burst neurons ( $\mathrm{rEBN}$ ) and a population of inhibitory short lead burst neurons (rIBN), and mirror symmetrically for the ILR [127, 128]. Firing patterns of EBN and IBN are similar. Collectively they are called short lead burst neurons (SBN). They are inhibited during fixation by omnipause neurons (OPN) (see [34]). The SBN encode linearly horizontal eye velocity and acceleration during the saccade, but with an earlier on- and offset than MN. For them the number of spikes in the burst encodes the change $\pm \Delta H$ of horizontal eye position:

$$
N=\int f(t) d t=a+b \cdot \Delta H+\text { noise }
$$

Below LR one found populations of burst-tonic neurons (BTN) with firing pattern similar to horizontal MN.

Robinson deduced from these firing patterns the neural integrator hypothesis: For conjugate horizontal saccades the SBN provide the pulse and pause in the MN, and their velocity signal is integrated $(1 / s$ on Laplace transforms and a direct input $\sim T)$ by the BTN to provide the step in the $\mathrm{MN}$ firing:

$$
\widetilde{I_{\mathrm{MN}}}(s)=(T+1 / s) \widetilde{I_{\mathrm{VEL}}}(s)
$$

He conjectured from (8), that there is a common neural integrator receiving velocity inputs for all conjugate horizontal eye movements, i.e. also for smooth pursuit and stabilization movements.

In good approximation, a temporal code holds in this scenario: All rightward saccades are driven by the population of $\mathrm{rSBN}$, and similarly by $\mathrm{ISBN}$ for leftward saccades, and their firing rate is proportional to $\pm d H / d t$ and $\pm d^{2} H / d t^{2}$. The same is true for the MN and the integrator neurons, with an additional eye position signal $H$. During rightward saccades the rEBN and rBTN excite the MN of the rLR and the IMR, the rIBN inhibit the ILR and rMR. Today one knows that the rEBN are disynaptically driven by saccade neurons in the left $\operatorname{motSC}$ (see [112]).

Input $\mathrm{RET} \rightarrow \mathrm{SC}:$ When the eyes are in forward direction, a visual direction from the right half-space is mapped by the optics of the eyes on the lateral hemi-retina of the left eye and the medial hemi-retina of the right eye. The output, from the former directly and from the latter after crossing the midline, projects to the left visSC and via the LG nucleus of TH to the left V1. If in this direction $\Phi$ is a target for a saccade, then the eye should 
according to (6) rotate by an $\Delta H=\Phi$ in order to foveate it. This could be accomplished, if in topographic map of visSC the neurons with visual RF at $\Phi$ activated the motor cells in the underlying motSC at $\Delta H=\Phi$, i.e. if the topographic map of the neurons in SC had a common coordinate $u$, which in visSC 'meant' $\Phi$ and in motSC $\Delta H$.

The crossing by the laws of optics is therefore compensated by the crossing of the fibers from the left motSC to the right EBN. This scheme is a beautiful example of an 'embodiment', that the structure of the external world is continued functionally accross the brain [87].

Robinson [96] and Schiller and Stryker [106] found that microstimulation in the SC evoked saccades, independent of initial eye position. These saccades were organized in a $2 \mathrm{D}$ topographic map, a smooth correspondence between the physical coordinates $(u, v)$ in SC and horizontal and vertical eye displacement $(\Delta H, \Delta V)$ of the stimulationinduced saccade. Since $(\Delta H, \Delta V)$ also describes the acquisition of a visual target which was sensed in the visSC above, the microstimulation data could also be interpreted as an afferent mapping from a hemiretina with polar coordinates $R$ (eccentricity in deg) and $\Phi$ (meridional target direction in deg) to SC coordinates $(u, v)$.

The torsional component of the saccades evoked by microstimulation was unknown in 1972. When 3D eye movement recordings became possible, two conflicting predictions emerged:

Let the eye be in an initial position $\boldsymbol{r}_{1}$ in Listing's plane and generate a saccade with end position $\boldsymbol{r}_{2}$ by microstimulation. The simplest hypothesis was, that the entire saccade trajectory $\boldsymbol{r}(t)$ remained in Listing's plane [49]. This would be true, if the saccaderelated burst neurons in motSC had movement fields in 2D eye displacement coordinates $(\Delta H, \Delta V)$. Tweed and Vilis [136] proposed that the SC encoded the motor error, the rotation $\boldsymbol{q}_{21}=\boldsymbol{r}_{2} \circ\left(-\boldsymbol{r}_{1}\right)$, which by (1) can have a torsional component, even for $\boldsymbol{r}_{1}$ and $\boldsymbol{r}_{2}$ in Listing's plane. Then different placements of the stimulation electrode in motSC could evoke saccades with $\mathbf{r}(t)$ not in Listing's plane.

The experiment by van Opstal et al. [143] showed that microstimulation induced saccades remained in Listing's plane, and Hepp et al. [51] found that the movement fields in motSC were 2D in eye displacement or (equivalently in the workspace of a $\mathrm{M} \Phi$ ) in retinal coordinates. Klier et al. [61] have shown that even for a head-free monkey in the wide range of natural gaze (eye and head) movements, motSC commands are in retinal coordinates. It was rather surprising to find a motor area operating in sensory coordinates. Clearly, deep in the brain, the distinction between 'sensory' and 'motor' becomes ambiguous.

In spite of many investigations major unsolved problems remain about the saccadic control by the SC:

- Do muscle pulleys contribute to implement Listing's law, since one expects in a temporal code that the SBN encode eye angular velocity $\omega(t)$, which can by (2) have a torsional component even for saccades in Listing's plane [22, 72]? However, such a signal has not been observed in $M \Phi$ 's perifoveal range [105].

- What is the biophysics of the integrator [1]?

- Where do the feedback loops close, which guarantee that visually evoked saccades reach their targets even after dramatic perturbations [58, 124, 140]?

Surprisingly, a simple mathematical model of a neural code in the motSC has recently been found:

Ottes et al. [85] chose SC coordinates $u$ to be the anatomical distance from the foveal representation in the SC measured along the horizontal meridian representation and $v$ to be the perpendicular anatomical distance. The best fit to the microstimulation data of [96] was a 
$\log$-polar map with the parameters $A, B_{u}$ and $B_{v}$ :

$$
\begin{aligned}
& u=B_{u} \ln \left[\left(R^{2}+2 A R \cos \Phi+A^{2}\right)^{1 / 2}\right] \\
& v=B_{v} \arctan [R \sin \Phi /(R \cos \Phi+A)]
\end{aligned}
$$

with the inverse in Listing coordinates, for $\Delta H=R \cos \Phi$ and $\Delta V=R \sin \Phi$,

$$
\Delta H(u, v)=A \cdot\left[\exp \left(u / B_{u}\right) \cdot \cos \left(v / B_{v}\right)-1\right], \quad \Delta V(u, v)=A \cdot \exp \left(u / B_{u}\right) \cdot \sin \left(v / B_{v}\right)
$$

Using $(13,14)$ it was a bold assumption by van Gisbergen et al. [141] to associate to every saccade neuron in motSC a spherically symmetric movement field with center $\left(u_{0}\right.$, $v_{0}$ ) on the SC motor map, by fitting the number $N$ of spikes in the burst, from $20 \mathrm{~ms}$ before saccade onset to $20 \mathrm{~ms}$ before end, to a truncated Gaussian distribution

$$
N(R, \Phi)=N_{0} \exp \left[-\frac{\left(u-u_{0}\right)^{2}+\left(v-v_{0}\right)^{2}}{2 \sigma_{0}^{2}}\right]
$$

This fit turned out to be very good and explained the distorted mountain found by [125] in Listing coordinates, related to $(u, v)$ by (15). $N_{0}$ and $\sigma_{0}^{2}$ showed no systematic relation with the SC motor map coordinates in motSC, except for very small saccades $[40,65]$. This indicates that for different saccade vectors the magnitude and spatial extent of the $\mathrm{SC}$ activation profile could be about the same. Assuming a uniform cell density across the SC motor map, the brainstem could receive from the motSC roughly the same number of spikes for every saccade. Marino et al. [65] performed the same analysis for the visual and the saccadic bursts in visuo-saccadic neurons in motSC and found that the visual receptive fields and the movement fields are approximately spherically symmetric with same center (in the SC coordinates $(u, v)$ ). The visual receptive fields were significantly smaller than the movement fields.

Goossens and van Opstal [40] recorded from a large number of neurons in motSC, which covered the motor map rather densely. They considered a lattice on the SC map and related a given saccade vector $(\Delta H, \Delta V)$ to the average spike activity $f_{i}(t)$ of the recorded neurons around the lattice sites $\left(u_{i}, v_{i}\right), 1 \leqslant i \leqslant M$. For this they assumed that every (average) neuron $i$ contributed a 'minivector'

$$
\boldsymbol{e}_{i}=\left(e_{i H}, e_{i V}\right)=\left(\gamma_{H} H\left(u_{i}, v_{i}\right), \gamma_{V} V\left(u_{i}, v_{i}\right)\right)
$$

and an input $\left(e_{i H} f_{i}(t-\tau), e_{i V} f_{i}(t-\tau)\right)$ to the innervation $\left(I_{H}(t), I_{V}(t)\right)$ which the motSC sends to the saccade generator, to generate a given saccade $(\Delta H, \Delta V)$, and that these 'minibursts' added linearly

$$
I_{H}(t)=\sum_{i=1}^{M} e_{i H} f_{i}(t-\tau), \quad I_{V}(t)=\sum_{i=1}^{M} e_{i V} f_{i}(t-\tau),
$$

Into $f_{i}(t)$ entered the measured times $t_{i, k}$, where the neuron $i$ fired in the burst under consideration, from $20 \mathrm{~ms}$ before saccade onset to $20 \mathrm{~ms}$ before saccade end. Then they calculated the trajectory of the saccade $\boldsymbol{r}(t)$ by feeding $I_{H}(t)$ and $I_{V}(t)$ into two identical versions of a linear temporal saccade generator composed of Robinson's linear plant model and of a linear integral feedback, inspired by the Scudder [111] model. The linear saccade generator consisted of Robinson's linear plant model (10) with the transfer function $(1+s T)^{-1}$, multiplied 
by the transfer function $\left(s^{-1}+T\right)$ for the integrator (12), and multiplied by $\left(s+\alpha e^{\beta s}\right) / \alpha$ for the linear integral feedback. Here the forward gain $\alpha$, the feedback delay $\beta$, the scaling parameters $\gamma_{H}$ and $\gamma_{V}$ in (17) and the time delay $\tau$ in (18) were fitted to generate best the $2 \mathrm{D}$ eye displacement and eye velocity profiles for a few saccades distributed evenly across the oculomotor range.

It was amazing, how well this dynamical decoding scheme reproduced the trajectory pattern of normal straight saccades, which exhibits many nonlinearities ('main sequence'): Saccades duration and peak velocity were proportional to amplitude, and the horizontal and vertical component trajectories of oblique saccades were stretched, so that the vector sum obeyed the main sequence rules (see Fig. 6 in [40]). The model also predicted the static movement fields, i.e. the measured number of spikes as function of amplitude and direction. Goossens and van Opstal ([38-40]) had additional data on blink perturbed visually evoked saccades. Here they could predict the progression of the eye from its starting point along the optimal saccade vector. The perturbed saccades could deviate strongly from a straight line and be considerably slowed down. Still, when the recorded data were analyzed in this scheme, they obeyed surprisingly well the theory.

This model should be taken as an 'existence proof' for a simple mathematical implementation of the space-time transformation between the RET, the SC and the trajectory of the eye, inspired by an abstraction of the downstream premotor circuits and the plant, for saccades in Listing's plane. However, it is only a decoding scheme. The predicted nonlinearities of the normal saccades were based entirely on the recorded population of saccade-related burst neurons in motSC, using separate trials for every single neuron. In the neurobiology literature, there are many 'ideal observer' schemes, where behavior can be decoded from measured neural activity (e.g. the fMRI-data of [122] on Free Will!). Can this scheme be a useful model for operations in the real brainstem? One possibility is that such a decoding scheme is used by the brain as part of an internal model to predict the saccade trajectories and their sensorimotor consequences from the distributed activity in motSC. More in Sect. 7. In the scheme by Goossens and van Opstal [40] one has to explain how the measured inputs $\left\{f_{i}(t)\right\}$ are generated by the input to motSC [142].

The visual burst in the visuo-saccadic neurons in motSC could be related to the activity in the upper layer, visSC, of the superior colliculus, which is directly driven by the retina. The visual RF in visSC are closely aligned with the saccadic fields in motSC [106]. For maintaining fixation during the permanent excitation by the retina in the light, inhibition between visSC and motSC is necessary, which could come from gating by the fixation neurons in the rostral motSC [76] and from the basal ganglia [52]. The ultimate gate are the OPN in the BS. However, much more intelligence than generated by the visSC is necessary for controlling cognitive saccades in M $\Phi$ 's activities, and this is provided by the neocortex, the topic of the next section.

It is sobering to realize that one week after the removal of one $\mathrm{SC}$, a macaque can make saccades into the contralateral hemifield as before, with only a longer reaction time (loss of 'express saccades') and a decrease in saccade frequency, when the visually guided saccades were not imposed by the experimenter [2]. Input from many areas the neocortex, in particular the FEF [108, 113], can trigger visually evoked saccades, which are executed by the intact temporal burst generator in BS after some synaptic reconfigurations. However, immediately after bilateral inactivation of the SC (by an injection of Muscimol, which reversibly enhances inhibition), the alert monkey behaves as if he were in the dark, with a low frequency of large resetting saccades, but without visual scanning of an interesting surround [51]. These symptoms remain permanent, if both the SC and the FEF are removed [107]. 


\section{Canonical Neocortical Circuit of the FEF}

Since we now 'understand' $\mathrm{M} \Phi$ at the level of the SC, we can turn to 'high level' visuomotor transformations in neocortex. We will start at the output from the FEF, since the input chain from V1 has not yet completely been worked out. Note, however, that V1 has in the perifoveal field a log-polar map, which is up to scaling identical to the SC map [110,133].

Again, we shall base our presentation on a bold hypothesis: The layered, local cortical circuit, which provides the anatomical substrate for all cortical computation, has been studied extensively in primary sensory cortex. These studies led to the hypothesis, that all areas of neocortex share a common 'canonical circuit' [27, 29, 30]. Using extensive anatomical reconstructions, Binzegger et al. [7] have cast their data into a recurrent network of four layers L2/3, L4, L5, and L6, for which they have obtained quantative data about numbers of excitatory and inhibitory neurons and their intra- and interlayer synaptic connections. This model is based on cat V1, but is conjectured to be generalizable to other cortical areas.

The FEF of the monkey is a functionally well studied area. Electrical stimulation [9, 99], recordings of thousands of neurons in the alert, behaving monkey (e.g. [8, 104, 131]) and lesion studies $[59,107]$ demonstrate that the FEF is a major cortical area for processing saccades (and for smooth pursuit in an adjacent area (see [62]). Visual responses and saccade vectors are topographically represented in the FEF [9]. Neurons in the FEF show responses related to visual saliency and selection, motor preparation, attention, working memory, and fixation, very similar to motSC, but less crisp in the discharge patterns of 'visual', 'visuosaccadic' and 'saccadic' neurons. The FEF has a direct projection to the BS saccade generator [108], which is normally overruled by the powerful direct pathway from the FEF layer 5 to motSC. In order to understand the logic of neocortex, we shall present the MBB model by Heinzle et al. [46] and show that the canonical circuit of Binzegger et al. [7] can, with a few modifications, explain basic operations of the primate FEF.

The control of saccades requires several computational steps: selection of a target, allocation of attention to the location of the intended target, and the motor output to the motSC that drives the eye movements. The selection of the target could follow a particular rule, as in an antisaccade task or in reading. Antisaccades are generated, when the subject responds to a visual stimulus with a saccade in the opposite direction [75]. In a simplified reading task the subject (saccadically) scans lines of words of different length and difficulty, and uses rules for word skipping and return at the end of a line [45, 47].

In addition, the oculomotor part of eye movement control interacts with cognitive processes that recognize visual features and influences how long attention is needed at a specific location. Such cognitive processes were not implemented in detail, but were condensed in a cortical module called REC (for recognition), which interacted with the FEF. The FEF model circuit received two external inputs: a retinotopic visual input, which represented the input from earlier visual areas, and a fixation input that was active when a fixation stimulus was present.

Layer 4 neurons received a feature unspecific visual input from early visual areas along the dorsal stream and selected the retinotopic position of the strongest of those inputs ([46], Fig 2). They formed a visual saliency map, with prospective capabilities for rapid scanning, so that a new stimulus was acquired as soon as attention was successfully allocated.

Layer 2/3 transformed the phasic signal of layer 4 into an attentional signal at the position of the selected target and stored it until the time of the saccade. This leaky neural integrator circuit was connected to the REC module and was activated by feature detection and recognition at the currently attended retinotopic position. Hence, they signaled the focus 
of attention. The REC module, in response, sent signals back to layer $2 / 3$ of the FEF when a target was 'recognized', as defined later. In addition, layer $2 / 3$ neurons drove the premotor neurons in layer 5 , which project topographically to the SC. Therefore, layer $2 / 3$ could be interpreted as both generating an attentional signal and a motor intention.

Layer 5 consisted of two functional types of neurons: build-up premotor neurons (L5r), which showed a ramping activity, and burst premotor neurons (L5b), which signaled the output to the SC and the brainstem. A population of fixation neurons inhibited the ramping activity in layer 5 .

Neurons in layer 6 projected back to layer 4 and biased the visual selection, or, under some conditions, they excited layer 4 in the absence of a visual input. They could provide a 'top-down saliency' signal that influenced the visual selection and could even induce a quasivisual signal in layer 4 , generated internally and looking like the response to a real visual input [68].

The attention-related top-down saliency was selected by a 'rule input' from the REC module to layer 6 . In saccades and during scanning, layer 6 did not receive a rule input. Then FEF ran in its 'default mode' in which the attentional signals in layer 6 were small and did not influence the selection of targets in layer 4. If the attended target had an antisaccade feature, the rule input targeted all retinotopic positions in layer 6 . This global input allowed layer 6 to be activated by its input from layer $2 / 3$ and hence achieve the remapping required for an antisaccade response. In 'no-go' trials, the rule input specifically targeted the foveal population of layer 6 . The top-down saliency depended on the action currently attended (through layer 6 as in antisaccade trials), but was also influenced by the last saccade, which induced an 'inhibition of return'.

The REC module consisted of three arrays of feature detectors for a saccade-, antisaccade- and no-go rule and an array of neurons expressing the recognition of the target ([46], Fig. S1). The feature detectors received a feature-specific visual input, which simulated input from the ventral processing pathways. Riesenhuber and Poggio [93] and Serre et al. [115] provide a possible implementation of such feature detectors in a feedforward network starting from V1. The REC module also uses maps of spiking neurons, but not as a layered neocortical circuit.

The canonical circuit, transplanted from the cat to the monkey, reproduced well the firing patterns of single neurons in FEF in different tasks and made testable predictions about unobserved neuronal populations. The model should be taken both as a plausibility proof and as a pedagogical tool. The above functional description shows how involved a realistic neural network is, in order to cope with neuroanatomy and with some of the many modes of visuo-motor transformations which the FEF generates. On the level of Blue Brain this is still impossible. Here one achieves some physiological realism by using anatomical data from cat V1 and 'integrate-and-fire' neurons. One obtained firing patterns corresponding well to those recorded in the monkey. The present implementation used more than 10.000 neurons and 2 Million synapses to represent 21 eye positions at $\{-10,-9, \ldots, 0, \ldots,+10\}$ with the fovea at 0 . The Matlab code runs on a laptop with a $4 \mathrm{GHz}$ Pentium processor, executing $1 \mathrm{~ms}$ real time in $1 \mathrm{sec}$. Instead of describing here all the results, the reader is encouraged to download the source code from the Web and play with it (www.ini.uzh.ch/ jakob/code/FEF_DEMO.zip).

This model does not simulate the full functionality of the FEF. No feedback from the SC is implemented, nor a perisaccadic remapping, as described in Sect. 7. Attention was just an intermediate step between vision and saccade, which is an overt shift of attention. About twice as fast (and sometimes required in society) are covert shifts of attention (i.e. without saccades), in which the FEF also plays an important role [131]. 
The influence of attention on VI neurons has been described in terms of its effect on the structure of RF, where multiple stimuli compete to drive neural responses and ultimately behavior. Armstrong et al. [4] stimulated the FEF of passively fixating monkeys and produced changes in V4 responses similar to those in voluntary attention: weak stimulation below the threshold for evoking saccades enhanced visual responses at particular locations within the RF. This altered the interaction between pairs of stimuli into the RF to favor those aligned with the activated FEF site. Thus spatial signals involved in saccade preparation are used to covertly select among multiple stimuli within the RF of VI neurons. Gotts et al. [41] recorded synchronous activity within and between areas V4 and FEF in attention. Neurons were chosen to have overlapping RF and the effects of attention in the joint RF were compared to attention outside. Attention led to increase in firing rate in both V4 and FEF, with earlier onset in FEF by $20-30 \mathrm{~ms}$, and to enhanced phase locking (at $\sim 40 \mathrm{~Hz}$ frequency) between electrodes within and across both areas. Phase locking was at about zero phase lag within V4 and FEF, but phase shifted by about $10 \mathrm{~ms}$ between FEF and V4, which may allow spikes emitted in one area to arrive at the time of maximal depolarization in the other area [36]. By this mechanism the postsynaptic influence of the attended stimulus could be enhanced compared to distractors. These results are consistent with a mechanism of attention in which prefrontal areas such as FEF guide visual selection through top-down biasing in extrastriate visual areas, leading to synchronous firing within and between regions as attention is sustained [116].

Similar to efforts in constructive quantum field theory it will be very revealing to study these phenomena in canonical cortical circuits.

\section{Translation Invariance}

Finally we reach recent experimental discoveries about the implementation of visual stabilty, which will lead us to neocortical networks implementing translation invariance by internal models.

If one taps on one side of one's eye with a finger-tip, the resulting image motion on the retina induces a strong perception of motion in the world. Von Helmholtz [146] knew that people suffering from eye paralysis reported jumps of the visual scene when they attempted to move their eyes, even though in this case the image on the retina remained stationary. He strongly argued for the importance of an 'effort of will', which in the second case is operative, but in the first not. The modern formulation by von Holst and Mittelstaedt [147] and Sperry [126] proposes that a corollary discharge (CD) of the motor command to the eye-ball is sent to the visual areas, to change the interpretation of the effect of the motor command or its absence. If the predicted and actual visual input differ, as in the finger tap, the discrepancy is interpreted as external motion (no $\mathrm{CD}$ ). If the eye is paralysed, the $\mathrm{CD}$ is generated, but there is no matching retinal shift (see e.g. [6]). Similar observations are now being made for arm movements in cerebellar patients [129].

Every saccade produces a shift of the visual image on RET. The RF of a visual neuron moves with the eye. In a seminal paper Duhamel et al. [31] found that single neurons in LIP are informed about intended saccades to update the representation of visual space. The intention to saccade evokes a transient shift in the retinal location at which the stimulus can excite the neuron. During fixation the RF is static. Immediately before and during the saccade the cortical representation shifts into the coordinates of the next intended fixation. Many LIP neurons have a forward field (FF), they transiently respond to stimuli at the next location and stop responding to stimuli at the initial location After the saccade the neuron 
responds again to the input from RET to its static RF. LIP neurons respond when the stimulus brings the site of a previously flashed stimulus in the RF, even if the stimulus is gone before the stimulus site enters their RF. If a flashed stimulus is carried out of the RF by a saccade, then its duration is shorter than in the normal decay. The majority of LIP neurons updates stimulus traces in multiple directions, for single neurons with different strength. At the population level remapping is independent of saccade direction [48]. This dynamic link between successive retinal images may contribute to the translation invariance of vision across saccades and to a continuous accurate retinocentric representation of the visual world.

Remapping has now been found to hold in all retinotopic areas along the dorsal visual stream and its output (FEF: [138, 139]; LIP: [13, 31, 48]; dorsal visual areas beyond V1: [77]) and in the SC [148]. Neurons transiently shift their static RF just before or during a saccade into the FF. A visual stimulus at the right time is necessary which by the saccade has to be carried into FF. If one equates 'spatial vision' with the visual response along the retinotopic dorsal pathway, then spatial vision should be stable across saccades. But in answer to the critique by Olshausen and Fields [84] this picture has to be considerably refined.

Sommer and Wurtz $[120,121]$ have investigated whether an internal copy of the motor command, which the SC sends to the oculomotor plant, is fed back to the FEF, to enable the perception of a stable visual world across saccades. While FEF projects directly to SC, the projection of SC to FEF has an intermediate link in the MD part of TH, through which the conjectured CD has to pass. Sommer and Wurtz [120] manipulated the position of the movement vector in the SC, which generated an aligned saccade vector in $\mathrm{MD}$, in relation to the position of the visual receptive field of a recorded neuron in FEF. By varying timing and receptive fields, they could show that the necessary spatio-temporal relations for a CD were satisfied. The crucial test was, when recording from well-isolated FEF neurons, that the spatial processing was lost during an reversible inactivation of MD. The result was that the visual processing of FEF neurons was spatiotemporally and functionally dependent on a CD in MD driven by SC. Two interpretations of these findings are plausible: On one hand the $\mathrm{CD}$ could be used to suppress vision during saccades, on the other hand the CD could provide the visual neurons in the FEF with a prediction of their sensory response after the saccade. The latter could be compared with the actual visual input, in order to determine changes in the external world. This would be a beautiful example of an internal model.

The same technique was used by Sommer and Wurtz $[118,119]$ to find significant deficits in double-step saccades [42] after interfering with the CD pathway $\mathrm{SC} \rightarrow \mathrm{MD} \rightarrow$ FEF. In this task two visual targets $s_{1}$ and $s_{2}$ are shown so rapidly one after the other, that $\boldsymbol{s}_{2}$ had disappeared, before the saccade to $\boldsymbol{s}_{1}$ had started. Then the first saccade vector $\boldsymbol{r}_{1}$ can be computed from $\boldsymbol{s}_{1}$ but the second, $\boldsymbol{r}_{2}-\boldsymbol{r}_{1}$, not from $\boldsymbol{s}_{2}$, but from the memory of $\boldsymbol{s}_{2}$ and a CD from the saccade $\boldsymbol{r}_{1}$, using Listing's law. The fact that the deficit was not complete shows that there are many parallel pathways operative (e.g. SC $\rightarrow \mathrm{MD} \rightarrow$ FEF and SC $\rightarrow$ PUL $\rightarrow$ LIP). Redundancy is the strength of a neural architecture.

The low capacity of short term memory does not allow to remap a visual image iconically across saccades $[55,91]$. However, such a detailed remapping is not necessary (see e.g. [71]). During the saccade the subject is effectively blind. Furthermore, focal visual attention is directed immediately before the saccade to the saccadic target and is carried by the saccade to its landing point [24]. This is effected by several neocortical operations, a presaccadic mapping in area V4 of the ventral stream towards the fovea [132] and an arbitration by IP [60]. After the saccade, the sensitivity of the visual pathways is enhanced $[37,74]$. For a fast interpretation of the postsaccadic image, it would be sufficient to remap 'pointers' to significant visual structures, and not entire maps (neuronal 'arrays'). This requires a mapping of intermediate level visual features in retinocentric coordinates. In the 
parietal area LIP, neurons with remapped retinocentric RF carry information about objects in space in terms of gain fields. Gain modulation is defined as a change in the sensitivity of a neural response to one set of inputs that depends on the activity of a second set of inputs. It was first observed in IP neurons that combine retinal and eye position signals in a multiplicative manner [3]. Gain field neurons and pointer maps have been modelled by Salinas and Abbott [102] and Hahnloser et al. [43]. The gain field principle [103] is used for coding cardinal numbers in LIP, as explained in the next section, where neural correlates of doing mathematical physics are analyzed.

In the high levels of the primate ventral visual stream neurons have large receptive fields selective to different objects (faces, houses, ...), but tolerant to changes in object position. For them transsaccadic remapping is not necessary, but a CD from the FEF could still be useful as a mechanim for learning position-tolerant object recognition. For, if the same image appears before and after a saccade in two different locations in the RF and if by a CD about the saccade vector the image is identified to arise from the same object, then this could contribute to an unsupervised learning of a translation-invariant representation of this object. In humans Cox et al. [15] swapped identity of two objects during saccades in such a manner that the new object appeared at the predicted position of the old one. They took advantage of the fact that during a saccade the subject was effectively blind and that the eye could be tracked in real time. They found that by 'breaking' of position invariance between two distinguishable, but similar objects, the visual system would associate both of them with the same object. Cox and Di Carlo [14] recorded in the MAC from single neurons in IT and found a significant un-learning effect after just one hour in the same paradigm, showing that rapid learning is possible in the mature NC. Transsaccadic remapping could reflect a mechanism by which the visual system builds and maintains tolerant object representations. Since FEF and IT are strongly interconnected, it would be interesting to inactivate the CD from SC to FEF while recording from 'confusable' neurons in IT.

Visual stability is, as we have seen, a complex theatrical performance. We might shudder, when we look at the backstage of our seemingly coherent visual world. The great Italian poet Montale [73] has described this experience:

Forse un mattino andando in un' aria di vetro / arida, rivolgendomi, vedrò compirsi il miracolo: / il nulla alle mie spalle, il vuoto dietro / di me, con un terrore di ubriaco.

Poi come s'uno schermo, s'accamperanno di gitto / alberi, case, colli per l'inganno consueto. / Ma sarà troppo tardi; ed io me n'andrò zitto / tra gli uomini che non si voltano, col mio segreto.

(My translation: Perhaps one morning, walking in a dry air of glass, / when turning around I will see the miracle happen: / the nothing at my shoulders, the emptiness behind me, / with the terror of a drunkard. / Then, as on a screen, arrange themselves suddenly / trees, houses and hills in the usual deception. / But it will be too late; and I will secretely walk / among the people which do not turn around, with my secret.)

\section{Doing Mathematical Physics in the Brain}

The philosopher Kant is one of the founding fathers of cognitive neuropsychology. By introspection he has gained important insights into the functioning of the human brain. Space has been considered by him as the 'form of the external sense', not in the Newtonian interpretation as a stage for physics, but also endowed with Euclidean geometry. In Sect. 17 of the 'Kritik der reinen Vernunft' Kant [57] he wrote: 
So ist die blosse Form der sinnlichen Anschauung, der Raum, noch gar keine Erkenntnis; er gibt nur das Mannigfaltige der Anschauung a priori zu einer möglichen Erkenntnis. Um aber irgend etwas im Raume zu erkennen, z.B. eine Linie, muss ich sie ziehen, und also eine bestimmte Verbindung des gegebenen Mannigfaltigen synthetisch zu Stande zu bringen, so, dass die Einheit dieser Handlung zugleich die Einheit des Bewusstseins (im Begriff einer Linie) ist, und dadurch allererst ein Objekt (ein bestimmter Raum) erkannt wird.

(Translation: Thus the mere form of outer sensible intuition, space, is not yet cognition at all; it only gives the manifold of intuition a priori for a possible cognition. But in order to cognize something in space, e.g. a line, I must draw it, and thus synthetically bring about a determinate combination of the given manifold, so that the unity of this action is at the same time the unity of consciousness (in the concept of a line), and thereby is an object (a determinate space) first cognized.)

Volontary saccades are objects in the sense of Kant, and so are saccadic polygons, e.g. triangles generated by visual targets in a double-step saccade. On the sensory side, numbers are important visual features, which the brain binds into abstract categories:

Human infants [56], Amazonian Indians [90] and monkeys [11] have a remarkable sense for numbers which is independent of language. At a glance the cardinality of a set can be assessed and is invariant with respect of other visual features. This is ecologically relevant for fighting or fleeing in the wilderness. The precision decreases with size according to Weber's law, $\Delta N / N=$ const. The laws for approximate addition and subtraction of children and apes are similar. There is increasing evidence for an automatic association of numbers with spatial positions. For instance, mental number bisections (e.g. reply to "what is halfway between two and ten?') are accompanied by a small leftward saccade, if a large number is followed by a small number, and less or in the opposite direction, if the large number followed the small number [64]. In the last decade the group of Dehaene has searched for neural correlates of numerosity in humans using fMRI [19, 53, 88, 89]. They found strong correlations in the intraparietal area VIP and lateral prefrontal (PF) cortical areas which confirm the psychological findings.

Recently Nieder and Miller and their collaborators have studied the same areas in the alert behaving macaque. They have found neuronal populations with labeled-line coding for cardinality, presently from 1 to 30 . Each neuron discharged most strongly for an optimal number $N$ and less for the neighbors. The width of the 'numerosity fields' increased with $N$ as in Weber's law. Nieder and his group made several important observations $[25,26$, 79-83, 134]: The onset of the activity was earlier in VIP than in PF. This is reasonable, since the numerosity neurons behave like visual filters, and VIP is closer to the primary visual cortex V1. The prefrontal number neurons seem to have a more 'cognitive' behaviour. They could be trained to associate their optimal cardinality $N$ with an abstract shape, for instance $N=7$ with Fig. 4 or the letter P. This is consistent with the role of prefrontal cortex in establishing semantic associations between signs and abstract categories, a precursor to symbolic thinking in linguistic humans. But abstract ordinal knowledge is also processed in IP [35] which is compatible with bidirectional connections between IP and PF. In LIP Roitman et al. [100] has found cardinality neurons of potentially an earlier stage, in which the discharge rate increased or decreased monotonically with $N$. These neurons have gain fields, since the retinal RF is multiplicatively modulated by numerosity in a monotonically ascending or descending manner. Furthermore their sensitivity to cardinality is much less the outcome of intensive training for numerosity. They fit well with models of cardinality as a feature of intermedate complexity between the visual array in the dorsal stream and cognitive representations in IP and PF [20, 145]. However, they could serve important purposes of their own depending on the task in which they are involved. 
A simple example for the usefulness of local numerosity is, when several groups of bad guys approach, to compute in what direction to prepare the escape. This we will take as the first model of a mathematical activity:

We introduce the LIP representation of local numerosity at places $\boldsymbol{s}_{1}, \ldots, \boldsymbol{s}_{n}$ as input to the microcircuit of the FEF (Sect. 6) and use the results in Sect. 7 on transsaccadic remapping by a CD from SC (Sect. 5). Then the following is a plausible a neural correlate for determining a weighted average of number locations

$$
\mathbf{S}=\sum N\left(s_{i}\right) \cdot s_{i}
$$

distibuted in clusters around $\boldsymbol{s}_{1}, \ldots, \boldsymbol{s}_{n}$ : The cardinality $N\left(\boldsymbol{s}_{i}\right)$ of the groups at $\boldsymbol{s}_{1}, \ldots, \boldsymbol{s}_{n}$ is extracted in the dorsal stream at LIP neurons with a monotonic representation. PF determines a saccade pattern to $\boldsymbol{s}_{i(1)}, \ldots, \boldsymbol{s}_{i(n)}$, for instance in the order $\boldsymbol{s}_{1}, \ldots, \boldsymbol{s}_{n}$, and this is executed by FEF. The first saccade is made to $\boldsymbol{s}_{1}$ and $N\left(s_{1}\right) \cdot \boldsymbol{s}_{1}$ is extracted from the gain field and stored into a 2D integrator (Working memory circuits have been found in PF). The FF for the other clusters are (centered around) $\boldsymbol{s}_{2}-\boldsymbol{s}_{1}, \ldots, \boldsymbol{s}_{n}-\boldsymbol{s}_{1}$ with cardinalities $N\left(\boldsymbol{s}_{2}\right), \ldots, N\left(\boldsymbol{s}_{n}\right)$, and these FF become the RF after $\boldsymbol{s}_{1}$ is performed. From Sect. 7 is clear that the counting process can proceed fast and smoothly. The remapping in LIP already starts after SC has decided to saccade and need not to wait until the eye actually moves. The content of the FF will become the content of the RF, after the eye has settled at the new position. The selection of the next target can also take place before the reading of the content $N\left(s_{1}\right)$ is terminated, and so on. One could implement algorithms of this type by a MBB model.

Next we speculate about a neural representation of a guided proof of Pythagoras' theorem. Let $A B C$ be (always in clockwise numbering) be a rectangular triangle with legs $B C$, $A C$ and hypotenuse $A B$, with length $|B C|=a,|A C|=b,|A B|=c$ and the right angle at $C$. The computer draws on the left side of the screen $A B C$, and exterior to $A B C$ squares $A C U V, C B X W, A E G B$ with surfaces $b^{2}, a^{2}, c^{2}$. This is the graphic representation of the assumptions leading to the claim that $c^{2}=a^{2}+b^{2}$ holds.

Now comes the help offered to $\mathrm{M} \Phi$ by the teacher: The computer imbeds $A B C$ in a square $C D F H$ with surface $|C D F H|=(a+b)^{2}$ with $A$ on $C D, E$ on $D F$ with $|D E|=b, G$ on $F H$ with $|F G|=b$ and $B$ on $H C$. On the right side of the screen the computer draws a square $C^{\prime} D^{\prime} F^{\prime} H^{\prime}$ with surface $(a+b)^{2}$ with $C^{\prime} D^{\prime}$ on the continuation of $C D$ beyond $D$. Hence $C^{\prime} H^{\prime}$ is parallel to $C H$, etc. There are additional points: $A^{\prime} \in C^{\prime} D^{\prime}, E^{\prime} \in D^{\prime} F^{\prime}, G^{\prime} \in F^{\prime} H^{\prime}, B^{\prime} \in C^{\prime} H^{\prime}$ with $\left|A^{\prime} D^{\prime}\right|=\left|D^{\prime} E^{\prime}\right|=\left|F^{\prime} G^{\prime}\right|=\left|B^{\prime} C^{\prime}\right|=b$. $A^{\prime} G^{\prime}$ intersects $B^{\prime} E^{\prime}$ orthogonally in a point $I^{\prime}, A^{\prime} D^{\prime} E^{\prime} I^{\prime}$ is a square with surface $b^{2}$ and $B^{\prime} I^{\prime} G^{\prime} H^{\prime}$ a square with surface $a^{2}$. Furthermore $A B C$ and $E^{\prime} G^{\prime} I^{\prime}, A D E$ and $C^{\prime} A^{\prime} I^{\prime}, E F G$ and $E^{\prime} F^{\prime} G^{\prime}, G H B$ and $B^{\prime} C^{\prime} I^{\prime}$ are pairwise shifted by a translation and they are all congruent with surface $a \cdot b / 2$.

$\mathrm{M} \Phi$ follows the rules of mathematical reasoning with the following neural implementation: In PF reside the axioms, the knowledge base of previous proofs and the steps for the logical order in the present proof. In IT the figures are represented and in FEF the shifts of the focus of attention. The proof operates on surfaces of triangles and squares, which $\mathrm{M} \Phi$ has learnt to be additive under cutting:

$$
\begin{aligned}
|C D F H| & =|A E G B|+|A B C|+|A D E|+|E F G|+|G H B| \\
\left|C^{\prime} D^{\prime} F^{\prime} H^{\prime}\right| & =\left|A^{\prime} D^{\prime} E^{\prime} I^{\prime}\right|+\left|B^{\prime} I^{\prime} G^{\prime} H^{\prime}\right|+\left|E^{\prime} G^{\prime} I^{\prime}\right|+\left|C^{\prime} A^{\prime} I^{\prime}\right|+\left|E^{\prime} F^{\prime} G^{\prime}\right|+\left|B^{\prime} C^{\prime} I^{\prime}\right|
\end{aligned}
$$

FEF and IT detect translated figures and deduce the equality of the following surfaces:

$$
|A C U V|=\left|A^{\prime} D^{\prime} E^{\prime} I^{\prime}\right|, \quad|C B X W|=\left|B^{\prime} I^{\prime} G^{\prime} H^{\prime}\right|
$$




$$
|C D F H|=\left|C^{\prime} D^{\prime} F^{\prime} H^{\prime}\right|, \quad|A B C|=\left|E^{\prime} G^{\prime} I^{\prime}\right|, \quad \ldots, \quad|G H B|=\left|B^{\prime} C^{\prime} I^{\prime}\right|
$$

Then M $\Phi$ 's PF applies the substitution and simplification rules for equations to finish the proof.

We expect that this algorithm can be implemented in a MBB model. Note that we did not rely on the full twodimensional Euclidean group $\mathbb{E}(2)$, but only on translations by saccades. Therefore Sects. 5,6 and 7 are relevant.

Using saccades $\mathrm{M} \Phi$ can draw and read figures. By learning a large data base of postulates and proven theorems he can follow his professor's presentation of the geometrical arguments in [33] on the screen, the blackboard of the teacher, and show his understanding by saccades (see e.g. [109]). He could take a course on the history of mechanics and follow the proof of the conservation of angular momentum for a mass point moving in a central force field geometrically as in ([78], Prop. I, Theorema I). Here he would use a sequence of saccades along the sequence of triangles in Newton's figure and might worry about the convergence of approximations. This form of doing mathematical physics, supervised by a teacher, is an unspectacular, but frequent activity. We do not yet understand the neural correlates of the higher levels of mathematical thinking, but we can image them in the scanner (see e.g. [63]).

The critical reader of this essay will complain, that we are grossly violating Robinson's dictum. We admit that we will never be certain that fine-grained neural representations of the algorithms, which we have sketchily outlined, are 'true' in a fundamental sense. However, it is encouraging that our understanding of the visuo-saccadic system including the prefrontal cortex PF, where the rules and the working memory reside [92, 130], is rapidly increasing. This one can see by browsing through the abstracts of the 2008 meeting of the Society for Neuroscience (www.sfn.org/am2008/).

We have started with saccades as a very powerful tool to direct overt attention. Processes in many cortical areas have to be synchronized in deploying attention [117]. Some of the imaging data are very suggestive for visualizing a $М \Phi$ doing mathematics, e.g. the binding of the ventral visual area IT for scrutiny, the hippocampus HI for memory and prefrontal cortex PF for rules, when a scrambled line-drawing has to be recognized [114]. In an overoptimistic interpretation of such fMRI and MEG activity we already approach the neural correlates of an EUREKA-experience, and might follow Dehaene and Changeux [21] to model mathematical insights as non-equilibrium phase transitions in a global neural workspace.

This could be the topic of another Festschrift in a decade.

\section{Conclusion}

We are far from understanding how we are 'doing' mathematical physics on our powerful analog brain computer. There is no threat that Blue Brain will simulate a mathematical physicist. In the future we will understand better how to read fMRI and the next generations of noninvasive images. We will put more and more electrodes into the human brain and create interfaces to machines and to other brains. We will enjoy great pleasures of discovery. But the neural correlates of abstract mathematics and creativity in theoretical physics can only be approached, when we understand on the neuronal level logic, poetry and consciousness.

Acknowledgements I am grateful to the late V. Henn for having introduced me to the brainstem, and to R.J. Douglas, J. Heinzle and K.A.C. Martin for having elevated me to the neocortex.

To Jürg Fröhlich I wish many happy and creative years in his beautiful Platonic world. 


\section{References}

1. Aksay, E., Olasagasti, I., Mensh, B.D., Baker, R., Goldman, M.S., Tank, D.: Functional dissection of circuitry in a neural integrator. Nature Neurosci. 10, 494-450 (2007)

2. Albano, J.E., Mishkin, M., Westbrook, L.E., Wurtz, R.H.: Visuomotor deficits following ablation of monkey superior colliculus. J. Neurophysiol. 48, 338-351 (1982)

3. Andersen, R.A., Essick, G.K., Siegel, R.M.: The encoding of spatial location by posterior parietal neurons. Science 320, 456-458 (1985)

4. Armstrong, K.M., Fitzgerald, J.K., Moore, T.: Changes in visual receptive fields with microstimulation of frontal cortex. Neuron 50, 791-798 (2006)

5. Bauby, J.-D.: Le Scaphandre et le Papillon. Laffont, Paris (1997)

6. Bays, P.M., Husain, M.: Spatial remapping of the visual world accross saccades. NeuroRep. 18, 12071213 (2007)

7. Binzegger, T., Douglas, R.J., Martin, K.A.C.: A quantitative map of the circuit of cat primary visual cortex. J. Neurosci. 24, 8441-8453 (2004)

8. Bruce, C.J., Goldberg, M.E.: Primate frontal eye fields. I. Single neurons discharging before saccades. J. Neurophysiol. 53, 603-635 (1985)

9. Bruce, C.J., Goldberg, M.E., Bushnell, M.C., Stanton, G.B.: Primate frontal eye fields. II. Physiological and anatomical correlates of electrically evoked eye movements. J. Neurophysiol. 54, 714-734 (1985)

10. Byers, W.: How Mathematicians Think. Princeton University Press, Princeton (2007)

11. Cantion, J.F., Brannon, E.M.: Basic math in monkeys and college students. PLoS Biol. 5, 2912-2919 (2007)

12. Changeux, J.-P., Connes, A.: Conversations on Mind, Matter, and Mathematics. Princeton University Press, Princeton (1998)

13. Colby, C.L., Goldberg, M.E.: Space and attention in parietal cortex. Ann. Rev. Neurosci. 22, 319-349 (1999)

14. Cox, D.D., Di Carlo, J.J.: Does learned shape selectivity in inferior temporal cortex automatically generalize across retinal position? J. Neurosci. 28, 10045-10055 (2008)

15. Cox, D.D., Meier, P., Oetelt, N., DiCarlo, J.J.: 'Breaking' position invariant object recognition. Nat. Neurosci. 8, 1145-1147 (2005)

16. Crawford, J.D.: Listing's law: what's all the hubbob? In: Harris, L.R., Jenkins, M. (eds.) Vision and Action. Cambridge University Press, Cambridge (1998)

17. Curcio, C.A., Sloan, K.R., Kalina, R.E., Hendrickson, A.E.: Human photoreceptor topography. J. Comput. Neurol. 292, 497-523 (1990)

18. Davis, P.J., Hersh, R.: The Mathematical Experience. Birkhäuser, Boston (1980)

19. Dehaene, S.: The Number Sense: How the Mind Creates Mathematics. Oxford University Press, London (1997)

20. Dehaene, S., Changeux, J.-P.: Development of elementary numerical abilities: a neuronal model. J. Cogn. Neurosci. 5, 390-407 (1993)

21. Dehaene, S., Changeux, J.-P.: Ongoing spontaneous activity controls access to consciousness: a model for inattentional blindness. PLoS Biol. 3, 910-927 (2005)

22. Demer, J.L., Kono, R., Wright, W.: Magnetic resonance imaging of human extraocular muscles in convergence. J. Neurophysiol. 89, 2072-2085 (2003)

23. Descartes, R.: Oeuvres et lettres, Pléiade. Gallimard, Paris (1953)

24. Deubel, H., Schneider, W.X.: Saccade target selection and object recognition: evidence for a common attentional mechanism. Vis. Res. 36, 1827-1837 (1996)

25. Diester, I., Nieder, A.: Semantic associations between signs and numerical categories in the prefrontal cortex. PLoS Biol. 5, 2684-2695 (2007)

26. Diester, I., Nieder, A.: Complementary contributions of prefrontal neuron classes in abstract numerical catagorization. J. Neurosci. 28, 7737-7747 (2008)

27. Douglas, R.J., Martin, K.A.C.: A functional microcircuit for cat visual cortex. J. Phys. (Lond.) 440, 735-769 (1991)

28. Douglas, R.J., Martin, K.A.C.: Neuronal circuits of the neocortex. Ann. Rev. Neurosci. 27, 419-451 (2004)

29. Douglas, R.J., Martin, K.A.C.: Mapping the matrix: the ways of neocortex. Neuron 56, 226-238 (2007)

30. Douglas, R.J., Martin, K.A.C., Witteridge, D.: A canonical microcircuit for neocortex. Neural Comput. 1, 480-488 (1989)

31. Duhamel, J.-R., Colby, C.L., Goldberg, M.E.: The updating of the representation of visual space in parietal cortex by intended eye movements. Science 255, 90-92 (1992)

32. Epshtein, B., Lifshitz, I., Ullman, S.: Image interpretation by a single bottom-up top-down cycle. Proc. Natl. Acad. Sci. USA 105, 14298-14303 (2008) 
33. Euclid ( -300) Euclid's Elements, Todhunter I. (ed.) Everyman's Library, London (1933)

34. Everling, S., Paré, M., Dorris, M.C., Munoz, D.P.: Comparison of the discharge characteristics of brain stem omnipause neurons and superior colliculus fixation neurons in monkey: implication for control of fixation and saccade behavior. J. Neurophysiol. 79, 511-528 (1998)

35. Fias, W., Lammertyn, J., Caessens, B., Orban, G.A.: Processing of abstract ordinal knowledge in the horizontal segment of the intraparietal sulcus. J. Neurosci. 27, 8952-8956 (2007)

36. Fries, P.: A mechanism for cognitive dynamics: neuronal communication through neuronal coherence. Trends. Cogn. Sci. 9, 474-480 (2005)

37. Gawne, T.J., Martin, J.M.: Response of primate visual cortical neurons to stimuli presented by flash, saccade, blink, and external darkening. J. Neurophysiol. 88, 2178-2186 (2002)

38. Goossens, H.H.L.M., van Opstal, A.J.: Blink-perturbed saccades in monkey. I. Behavioral analysis. J. Neurophysiol. 83, 3411-3429 (2000)

39. Goossens, H.H.L.M., van Opstal, A.J.: Blink-perturbed saccades in monkey. II. Superior colliculus activity. J. Neurophysiol. 83, 3430-3452 (2000)

40. Goossens, H.H.L.M., Van Opstal, A.J.: Dynamic ensemble coding of saccades in the monkey superior colliculus. J. Neurophysiol. 95, 2326-2341 (2006)

41. Gotts, S.J., Gregoriou, G.G., Zhou, H., Desimone, R.: Synchronous activity within and between areas V4 and FEF in attention. Neuroscience Meeting, Atlanta \#703.7 (2006)

42. Guthrie, B.L., Porter, J.D., Sparks, D.L.: Corollary discharge provides accurate eye position information to the oculomotor system. Science 221, 1193-1195 (1983)

43. Hahnloser, R., Douglas, R.J., Mahowald, M., Hepp, K.: Feedback interactions between neuronal pointers and maps for attentional processing. Nature Neurosci. 2, 746-752 (1999)

44. Haslwanter, T., Straumann, D., Hepp, K., Hess, B.J.M., Henn, V.: Smooth pursuit eye movements obey Listing's law in the monkey. Exp. Brain Res. 87, 470-872 (1991)

45. Heinzle, J.: A model of the local cortical circuit of the frontal eye fields. Diss ETHZ No. 16897 (2006)

46. Heinzle, J., Hepp, K., Martin, K.A.C.: A microcircuit model of the frontal eye fields. J. Neurosci. 27, 9341-9353 (2007)

47. Heinzle, J., Hepp, K., Martin, K.A.C.: A biologically realistic cortical model of eye movement control in reading. Submitted (2009)

48. Heiser, L.M., Colby, C.C.: Spatial updating in area LIP is independent of saccade direction. J. Neurophysiol. 95, 2751-2767 (2006)

49. Hepp, K.: On Listing's law. Commun. Math. Phys. 132, 285-292 (1990)

50. Hepp, K.: Theoretical explanations of Listing's law and their implication for binocular vision. Vis. Res. 35, 3237-3242 (1995)

51. Hepp, K., van Opstal, A.J., Straumann, D., Hess, B.J.M., Henn, V.: Monkey superior colliculus represents rapid eye movements in a two-dimensional motor map. J. Neurophysiol. 69, 965-979 (1993)

52. Hikosaka, O., Takikawa, Y., Kawagoe, R.: Role of the basal ganglia in the control of purposive saccadic eye movements. Phys. Rev. 80, 953-978 (2000)

53. Hubbard, E.M., Piazza, M., Pinel, P., Dehaene, S.: Interactions between number and space in parietal cortex. Nature Rev. Neurosci. 6, 435-448 (2005)

54. Hubel, D.H., Wiesel, T.N.: Functional architecture of macaque visual cortex. Proc. R. Soc. Lond. B 198, 1-59 (1977)

55. Irwin, D.E.: Information integration across saccadic eye movements. Cogn. Psychol. 23, 420-456 (1991)

56. Izard, D., Dehaene-Lambertz, G., Dehaene, S.: Distinct cerebral pathways for object identity and number in human infants. PLoS Biol. 6, 275-285 (2008)

57. Kant, I.: Kritik der reinen Vernunft. Suhrkamp, Frankfurt (1787) (Weischedel, W., ed.). English translation by Guyer P., Wood A.W.: Critique of Pure Reason. Cambridge University Press, Cambridge (1998)

58. Kato, R., Grantyn, A., Dalezios, Y., Moschovakis, A.K.: The local loop of the saccadic system closes downstream of the superior colliculus. Neuroscience 143, 319-337 (2006)

59. Keller, E.L., Lee, K.-M., Park, S.-W., Hill, J.A.: The effect of inactivation of the cortical frontal eye field on saccades generated in a choice-response paradigm. J. Neurophysiol. 100, 2726-2737 (2008)

60. Khan, A.Z., Blangero, A., Rosetti, Y., Salemme, R., Luauté, J., Deubel, H., Schneider, W.X., Laverdure, N., Rode, G., Boisson, D., Pisella, L.: Parietal damage dissociates saccade planning from presaccadic perceptual facilitation. Cereb. Cortex 19, 383-387 (2009)

61. Klier, E.M., Wang, H., Crawford, J.D.: The superior colliculus encodes gaze commands in retinal coordinates. Nature Neurosci. 4, 627-632 (2001)

62. Krauzlis, R.J.: Recasting the smooth pursuit eye movement system. J. Neurophysiol. 91, 591-603 (2004) 
63. Krueger, F., Scampinato, M.V., Pardini, M., Pajevic, S., Wood, J.N., Weiss, G.H., Landgraf, S., Grafman, J.: Integral calculus problem solving: an fMRI investigation. NeuroReport 19, 1095-1099 (2008)

64. Loetscher, T., Bockisch, C.J., Brugger, P.: Looking for the answer: the mind's eye in number space. Neuroscience 151, 725-729 (2008)

65. Marino, R.A., Rodgers, C.K., Levy, R., Munoz, D.P.: The spatial representation of visuomotor transformations in the superior colliculus. J. Neurophysiol. 100, 2564-2576 (2008)

66. Markram, H.: The blue brain project. Nature Rev. Neurosci. 7, 153-160 (2006)

67. Marr, D.: Vision. Freeman, San Francisco (1982)

68. Mays, L.E., Sparks, D.L.: Dissociation of visual and saccade-related responses in superior colliculus neurons. J. Neurophysiol. 43, 207-232 (1980)

69. McPeek, R.M.: Incomplete suppression of distractor-related activity in the frontal eye field results in curved saccades. J. Neurophysiol. 96, 2699-2711 (2006)

70. McPeek, R.M., Han, J.H., Keller, E.L.: Competition between saccade goals in the superior colliculus produces saccade curvature. J. Neurophysiol. 89, 2577-2590 (2003)

71. Melcher, D., Colby, C.L.: Trans-saccadic perception. Trends Cogn. Sci. 12, 466-473 (2008)

72. Miller, J.M., Robins, D.: Extraocular muscle sideslip and orbital geometry in monkeys. Vis. Res. 27, 381-392 (1987)

73. Montale, E.: Tutte le poesie. Montadori, Milano (1977)

74. Müller, J.R., Mehta, A.B., Krauskopf, J., Lennie, P.: Information conveyed by onset transients in responses of striate cortical neurons. J. Neurosci. 21, 6978-6990 (2001)

75. Munoz, D.P., Everling, S.: Look away: the antisaccade task and the volontary control of movement. Nature Rev. Neurosci. 5, 218-228 (2004)

76. Munoz, D.P., Wurtz, R.H.: Fixation cells in monkey superior colliculus I. Characteristics of cell discharge. J. Neurophysiol. 70, 559-575 (1993)

77. Nakamura, K., Colby, C.L.: Updating of the visual representation in monkey striate and extrastriate cortex during saccades. Proc. Natl. Acad. Sci. USA 99, 4026-4031 (2002)

78. Newton, I.: Philosophiae Naturalis Principia Mathematica. Pepys, London (1687)

79. Nieder, A.: Counting on neurons: the neurobiology of numerical competence. Nature Rev. Neurosci. 6, 177-190 (2005)

80. Nieder, A., Diester, I., Tudusciuc, O.: Temporal and spatial enumeration processes in the primate parietal cortex. Science 313, 1431-1435 (2006)

81. Nieder, A., Merten, K.: A labeled-line code for small and large numerosities in the monkey prefrontal cortex. J. Neurosci. 27, 5986-5993 (2007)

82. Nieder, A., Miller, E.K.: Coding of cognitive magnitude: compressed scaling of numerical information in the primate prefrontal cortex. Neuron 37, 149-157 (2003)

83. Nieder, A., Miller, E.K.: A parieto-frontal network for visual numerical information in the monkey. Proc. Natl. Acad. Sci. USA 101, 7457-7462 (2004)

84. Olshausen, B.A., Field, D.J.: How close are we to understanding V1? Neural Comput. 17, 1665-1699 (2005)

85. Ottes, F.P., van Gisbergen, J.A.M., Eggermont, J.J.: Visuomotor fields of the superior colliculus: a quantitative model. Vis. Res. 26, 857-873 (1986)

86. Penrose, R.: Shadows of the Mind. Oxford University Press, New York (1995)

87. Pfeifer, R., Lungarella, M., Iida, F.: Self-organization, embodiment, and biologically inspired robotics. Science 318, 1088-1093 (2007)

88. Piazza, M., Izard, V., Pinel, P., Le Bihan, D., Dehaene, S.: Tuning curves for approximate numerosity in the human intraparietal sulcus. Neuron 44, 547-555 (2004)

89. Piazza, M., Pinel, P., Le Bihan, D., Dehaene, S.: A magnitude code common to numerosities and number symbols in human intraparietal cortex. Neuron 53, 293-305 (2007)

90. Pica, P., Lemer, C., Izard, V., Dehaene, S.: Exact and approximate arithmetic in a Amazonian indigene group. Science 306, 499-503 (2004)

91. Prime, S.L., Tsotsos, L., Keith, G.P., Crawford, J.D.: Visual memory capacity in transsaccadic integration. Exp. Brain Res. 180, 609-628 (2007)

92. Rao, S.C., Rainer, G., Miller, E.K.: Integration of what and where in the primate prefrontal cortex. Science 276, 821-824 (1997)

93. Riesenhuber, M., Poggio, T.: Hierarchical models of object recognition in cortex. Nature Neurosci. 2, 1019-1025 (1999)

94. Robinson, D.A.: A method of measuring eye movements using a scleral search coil in a magnetic field. IEEE Trans. Biomed. Eng. 10, 137-145 (1963)

95. Robinson, D.A.: Oculomotor unit behavior in the monkey. J. Neurophysiol. 33, 393-404 (1970)

96. Robinson, D.A.: Eye movements evoked by collicular stimulation in the alert monkey. Vis. Res. 12, 1795-1808 (1981) 
97. Robinson, D.A.: The use of control systems analysis in the neurophysiology of eye movements. Ann. Rev. Neurosci. 4, 463-503 (1981)

98. Robinson, D.A.: Implications of neural networks for how we think about brain function. Behav. Brain Sci. 15, 644-553 (1992)

99. Robinson, D.A., Fuchs, A.F.: Eye movements evoked by stimulation of frontal eye fields. J. Neurophysiol. 32, 637-648 (1969)

100. Roitman, J.D., Brannon, E.M., Platt, M.L.: Monotonic coding of numerosity in macaque lateral intraparietal area. PLoS Biol. 5, 1672-1682 (2007)

101. Ruelle, D.: The Mathematician's Brain. Princeton University Press, Princeton (2007)

102. Salinas, E., Abbott, L.F.: A model of multiplicative neural responses in parietal cortex. Proc. Natl. Acad. Sci. USA 93, 11956-11961 (1997)

103. Salinas, E., Thier, P.: Gain modulation: a major computational principle of the central nervous system. Neuron 27, 15-21 (2000)

104. Sato, T.R., Schall, J.D.: Effects of stimulus-response compatibility on neural selection in frontal eye field. Neuron 38, 637-648 (2003)

105. Scherberger, H., Cabungcal, J.-H., Hepp, K., Suzuki, Y., Straumann, D., Henn, V.: Ocular counterroll modulates the preferred direction of saccade-related burst neurons in the monkey. J. Neurophysiol. 86, 935-493 (2001)

106. Schiller, P., Stryker, M.: Single-unit recording and stimulation in superior colliculus of the alert rhesus monkey. J. Neurophysiol. 35, 915-924 (1972)

107. Schiller, P.H., True, S.D., Conway, J.L.: Deficits in eye movements following frontal eye field and superior colliculus ablations. J. Neurophysiol. 44, 1175-1189 (1980)

108. Schnyder, H., Reisine, H., Hepp, K., Henn, V.: Frontal eye field projection to the paramedian pontine reticular formation traced with wheat germ agglutinin in the monkey. Brain Res. 329, 151-160 (1985)

109. Schultz, J.E., Hallowell, K.A., Ellis Jr., W., Kennedy, P.A., Engelbrecht, M., Rutkowsky, K.: Geometry. Holt, Rinehart \& Winston, Austin (2001)

110. Schwartz, E.L.: Computational anatomy and functional architecture of striate cortex: A spatial mapping approach to perceptual coding. Vis. Res. 20, 645-669 (1980)

111. Scudder, C.A.: A new local feedback model of the saccadic burst generator. J. Neurophysiol. 59, 14551475 (1988)

112. Scudder, C.A., Kaneko, C.R.S., Fuchs, A.F.: The brainstem burst generator for saccadic eye movements. A modern synthesis. Exp. Brain Res. 142, 439-462 (2002)

113. Segraves, M.A.: Activity of monkey frontal eye field neurons projecting to oculomotor regions of the pons. J. Neurophysiol. 68, 1967-1985 (1992)

114. Sehatpour, P., Molholm, S., Schwartz, T.H., Mahoney, J.R., Mehta, A.D., Javitt, J.C., Stanton, P.K., Foxe, J.J.: A human intracranial study of long-range oscillatory coherence across a frontal-occipitalhippocampal brain network during visual object processing. Proc. Natl. Acad. Sci. USA 105, 43994404 (2008)

115. Serre, T., Oliva, A., Poggio, T.: A feedforward architecture accounts for rapid categorization. Proc. Natl. Acad. Sci. USA 104, 6424-6429 (2007)

116. Siegel, M., Donner, T.H., Oostenveld, R., Fries, P., Engel, A.K.: Neuronal synchronization along the dorsal visual pathway reflects the focus of spatial attention. Neuron 60, 709-719 (2008)

117. Singer, W., Gray, C.M.: Visual feature integration and the temporal correlation hypothesis. Ann. Rev. Neurosci. 18, 555-586 (1995)

118. Sommer, M.A., Wurtz, R.H.: What the brainstem tells the frontal cortex. II. Role of the SC-MD-FEF pathway in corollary discharge. J. Neurophysiol. 91, 1403-1423 (2004)

119. Sommer, M.A., Wurtz, R.H.: What the brainstem tells the frontal cortex. I. Oculomotor signals sent from superior colliculus to frontal eye field via mediodorsal thalamus. J. Neurophysiol. 91, 1381-1402 (2004)

120. Sommer, M.A., Wurtz, R.H.: Influence of the thalamus on spatial visual processing in frontal cortex. Nature 444, 374-377 (2006)

121. Sommer, M.A., Wurtz, R.H.: Brain circuits for the internal monitoring of movements. Ann. Rev. Neurosci. 31, 317-338 (2008)

122. Soon, C.S., Brass, M., Heinze, H.J., Haynes, J.D.: Unconscious determinants of free decisions in the human brain. Nature Neurosci. 11, 543-45 (2008)

123. Sparks, D.L.: Translation of sensory signals into commands for the control of saccadic eye movements: role of primate superior colliculus. Physiol. Rev. 66, 118-171 (1986)

124. Sparks, D.L.: The brainstem control of saccadic eye movements. Nature Rev. Neurosci. 3, 952-964 (2002)

125. Sparks, D.L., Mays, L.E.: Movement fields of saccade-ralated burst neurons in monkey superior colliculus. Brain Res. 190, 39-50 (1980) 
126. Sperry, R.W.: Neural basis of the spontaneous optokinetic response produced by visual inversion. J. Comput. Physiol. Psychol. 43, 482-489 (1950)

127. Strassman, A., Highstein, S.M., McCrea, R.A.: Anatomy and physiology of saccadic burst neurons in the alert squirrel monkey. I. Excitatory burst neurons. J. Comput. Neurol. 249, 337-357 (1986)

128. Strassman, A., Highstein, S.M., McCrea, R.A.: Anatomy and physiology of saccadic burst neurons in the alert squirrel monkey. II. Inhibitory burst neurons. J. Comput. Neurol. 249, 358-380 (1986)

129. Synofzik, M., Lindner, A., Thier, P.: The cerebellum updates predictions about the visual consequences of one's behavior. Current Biol. 18, 814-818 (2008)

130. Tanji, J., Hoshi, E.: Role of the lateral prefrontal cortex in executive behavioral control. Physiol. Rev. 88, 37-57 (2008)

131. Thompson, K.G., Biscoe, K.L., Sato, T.R.: Neuronal basis of covert spatial attention in the frontal eye field. J. Neurosci. 25, 9479-9487 (2005)

132. Tolias, A.S., Moore, T., Smirnakis, S.M., Tehovnik, E.J., Siapas, A.G., Schiller, P.J.: Eye movements modlate visual receptive fields of V4 neurons. Neuron 29, 757-767 (2001)

133. Tootell, R.B.H., Switkes, E., Silverman, M.S., Hamilton, S.L.: Functional anatomy of macaque striate cortex. II. Retinotopic organization. J. Neurosci. 8, 1531-1568 (1988)

134. Tudusciuc, O., Nieder, A.: Neuronal population coding of continuous and discrete quantity in the primate posterior parietal cortex. PNAS 104, 14513-14518 (2007)

135. Tweed, D.: Visual-motor optimization in binocular control. Vis. Res. 37, 1939-1951 (1997)

136. Tweed, D., Vilis, T.: The superior colliculus and spatiotemporal translation in the saccadic system. Neural Netw. 3, 75-86 (1990)

137. Tweed, D., Fetter, M., Anreadaki, S., Koenig, E., Dichgans, J.: Three-dimensional properties of human pursuit eye movements. Vis. Res. 32, 1225-1238 (1992)

138. Umeno, M.M., Goldberg, M.E.: Spatial processing in the monkey frontal eye field. I. Predictive visual responses. J. Neurophysiol. 78, 1373-1383 (1997)

139. Umeno, M.M., Goldberg, M.E.: Spatial processing in the monkey frontal eye field. II. Memory responses. J. Neurophysiol. 86, 2344-2352 (2001)

140. van Gisbergen, J.A.M., Robinson, D.A., Gielen, S.: A quantitative analysis of generation of saccadic eye movements by burst neurons. J. Neurophysiol. 45, 417-442 (1981)

141. van Gisbergen, J.A.M., van Opstal, A.J., Tax, A.A.M.: Collicular ensemble coding of saccades based on vector summation. Neuroscience 21, 541-555 (1987)

142. van Opstal, A.J., Goossens, H.H.L.M.: Linear ensemble-coding in midbrain superior colliculus specifies the saccade kinematics. Biol. Cybern. 98, 561-577 (2008)

143. van Opstal, A.J., Hepp, K., Hess, B.J.M., Straumann, D., Henn, V.: Two- rather than three-dimensional representation of saccades in monkey superior colliculus. Science 252, 1313-1315 (1991)

144. van Opstal, A.J., Hepp, K., Suzuki, Y., Henn, V.: Role of monkey nucleus reticularis tegmenti pontis in the stabilization of Listing's plane. J. Neurosci. 15, 7284-7296 (1996)

145. Verguts, T., Fias, W.: Representation of number in animals and humans: a neural model. J. Cogn. Neurosci. 16, 1493-1504 (2004)

146. von Helmholtz, H.: Handbuch der Physiologischen Optik. Voss, Leipzig (1867)

147. von Holst, E., Mittelstaedt, H.: Das Reafferenzprinzip. Wechselwirkungen zwischen Centralnervensystem und Peripherie. Naturwissenschaften 37, 464-476 (1950)

148. Walker, M.F., Fitzgibbon, E.J., Goldberg, M.E.: Neurons in the monkey superior colliculus predict the visual result of impending saccadic eye movements. J. Neurophysiol. 73, 1988-2003 (1995) 